\title{
Etude électrophysiologique préliminaire de la régénération du nerf alvéolaire inférieur
}

\section{Preliminary electrophysiological study of inferior alveolar nerve regeneration}

\author{
ROZÉ DAVID*, LIBERSA PHILIPPE*, LIBERSA JEAN-CLAUDE*, PICQUET FLORENCE**
}

\begin{abstract}
RÉSUMÉ
Un protocole expérimental a été mené sur 8 lapins afin d'évaluer la régénération du nerf alvéolaire inférieur (IAN) 6 mois après section complète et suture conventionnelle. Le risque de lésions nerveuses pouvant survenir à la suite de certains actes de chirurgie buccale, comme les extractions de dents de sagesse mandibulaires, reste trop souvent méconnu. Pourtant dans $0,05 \%$ des cas, il existe à une lésion irréversible qui pourrait justifier une microsuture nerveuse.

Dans cette étude, des tests électrophysiologiques ont été réalisés à $\mathrm{J}+180$ pour quantifier de façon objective la régénération du nerf. Une étude histologique a permis de comparer structurellement mais aussi quantitativement les fibres nerveuses saines et celles remaniées. Les résultats obtenus montrent une régénération fonctionnelle dans 7 cas traités. (Med Buccale Chir Buccale 2003; 9: 21-27)
\end{abstract}

médecine buccale chirurgie buccale

VOL. $9, \mathrm{~N}^{\circ} 1$ 2003

page 21

mots clés : Nerf alvéolaire inférieur, Lapin, Suture nerveuse

\section{SUMMARY}

Nine adult rabbits were used for an experimental study. The aim of this study was to demontrate the reality of inferior alveolar nerve (IAN) 6 months after total section and conventional suture.

IAN damage can occur in oral surgical procedures like third molar removal. However, 0,05\% of cases needs an microsurgical approach of the lesion.

In this preliminary study, electrical stimulation and histological study of the nerve was performed to record electrophysiological activity and compared different fibres.

24 weeks's results showed the possibility of nerve regeneration in 7 rabbits, and pointed out the decrease in half of nervous fibres. (Med Buccale Chir Buccale 2003; 9: 21-27)

key words : Inferior alveolar nerve, Rabbit, Nerve suture

\footnotetext{
* Centre Abel Caumartin, CHRU Lille. Lauréat de la bourse de recherche de la SFMBCB.

** Laboratoire de Plasticité Neuromusculaire, USTL

Demande de tirés à part :
}

Docteur D. Rozé Service d'Odontologie Centre Abel Caumartin CHRU Lille 59000 Lille 
médecine

buccale

chirurgie

buccale

VOL. $9, \mathrm{~N}^{\circ} 1$ 2003

page 22
De nombreux actes de chirurgie buccale peuvent conduire à une lésion iatrogène du nerf alvéolaire inférieur (NAl) : l'énucléation de kystes ou de tumeurs [1], l'intervention de chirurgie orthognatique $[2,3,4]$, les traitements endodontiques [4], les interventions de chirurgie implantaire [5], mais également et surtout l'avulsion de la troisième molaire mandibulaire $[6,7,8,9]$. Ainsi, il apparaît qu'une microsuture nerveuse pourrait être nécessaire dans $1 \%$ des cas de traumatismes iatrogènes. Sur 1000000 de dents de sagesse mandibulaires incluses extraites, on suspecte 50000 lésions nerveuses. Dans la grande majorité, le traumatisme est réversible (compression, section partielle) mais la section est totale dans $1 \%$ des cas [10]. II en résulte une perte de sensibilité des dents, de la gencive, de la lèvre inférieure et d'une partie du territoire cutané adjacent [11]. II a d'autre part été démontré que les dysesthésies après section nerveuse complète sont 18 fois plus importante lorsqu'il y a abstention thérapeutique au lieu d'effectuer une microsuture [12]. Dans cette étude, les auteurs se proposent de mettre en place une technique fiable et reproductible de suture nerveuse sur le lapin après section complète du NAI. La régénération objective du NAl est contrôlée par des tests électrophysiologiques capable d'enregistrer des potentiels nerveux globaux sous forme de potentiels évoqués par stimulation électrique. Les résultats sont associés à une analyse histologique comparative des fibres nerveuses saines et remaniées.

\section{MATÉRIEL ET MÉTHODES}

Dans cette étude préliminaire, 8 lapins adultes femelles - New Zealand Conventionnel SEGAV de $3,5 \mathrm{~kg}$ - ont été utilisés. L' expérimentation animale a été réalisée avec l'autorisation du Ministère de l'Agriculture et de la Forêt (Service Vétérinaire de la Santé et de la protection des Animaux, autorisation $n^{\circ} 5900$ 980) et celle du Ministère de l'Education Nationale.

L'anesthésie a été obtenue à l'aide d'une solution composée de pentobarbital $(30 \mathrm{mg} / \mathrm{kg}$ en intrapéritonéal). Les poils situés sur l'hémiface inférieure gauche du lapin ont été rasés. Afin de la protéger, l'artère faciale était repérée par inspection et palpation de la région.

Une incision sous-mandibulaire, rectiligne, postéro-antérieure était faite au contact osseux sur une distance de $30 \mathrm{~mm}$ avec un bistouri conventionnel (lame 15).

Puis, les tissus sous-peauciers étaient réclinés afin de mettre en évidence la corticale mandibulaire dans la région du trou mentonnier. Afin de dégager une portion nerveuse suffisante pour réaliser une suture conventionnelle, une fenêtre osseuse aboutissant à un élargissement du trou mentonnier dans sa zone postéro-inférieure était réalisée. Pour réaliser la fenêtre osseuse, des micro-perforations corticales en timbre poste ont été effectuées à l'aide d'une fraise boule montée sur pièce à main sous irrigation continue au sérum physiologique. Les perforations étaient ensuite reliées entre elles avec une fraise Zékria à os montée sur un contre-angle bague rouge. Pendant le fraisage, le paquet vasculo-nerveux était toujours protégé par une spatule extra-fine et plate, dont l'extrémité était insérée dans le canal. Le volet osseux était alors être facilement détaché grâce à une micro-pince gouge. Le NAl était ensuite séparé de son artère homologue en interposant un champ bleu synthétique, imperméable et stérile, de $5 \mathrm{~mm}$ de largeur sur $10 \mathrm{~mm}$ de longueur (Fig. 1).

Le nerf était isolé des différents manchons fibreux et musculaires qui peuvent être à l'origine d'artéfact sur les tracés électrophysiologiques. Après avoir isolé le nerf, une première électrode, bipolaire, de stimulation, était mise en place en aval de la zone de section; puis une deuxième électrode, monopolaire, était implantée en amont de cette zone afin d'enregistrer l'influx nerveux (Fig. 1). Les électrodes étaient constitués d'argent pur et avaient un diamètre de $0,2 \mathrm{~mm}$. La distance entre l'électrode de stimulation et celle de réception était de $5 \mathrm{~mm}$. Des stimulations électriques d'une durée de $0,1 \mathrm{msec}$ étaient déclenchées afin d'enregistrer des potentiels nerveux globaux de référence. Dans un premier temps, on a cherché à déterminer l'intensité supraliminaire. 
Figure 1 : Mise en place des électrodes de stimulation et de réception (DR)

Stimulation and reference electrodes inserted (DR)

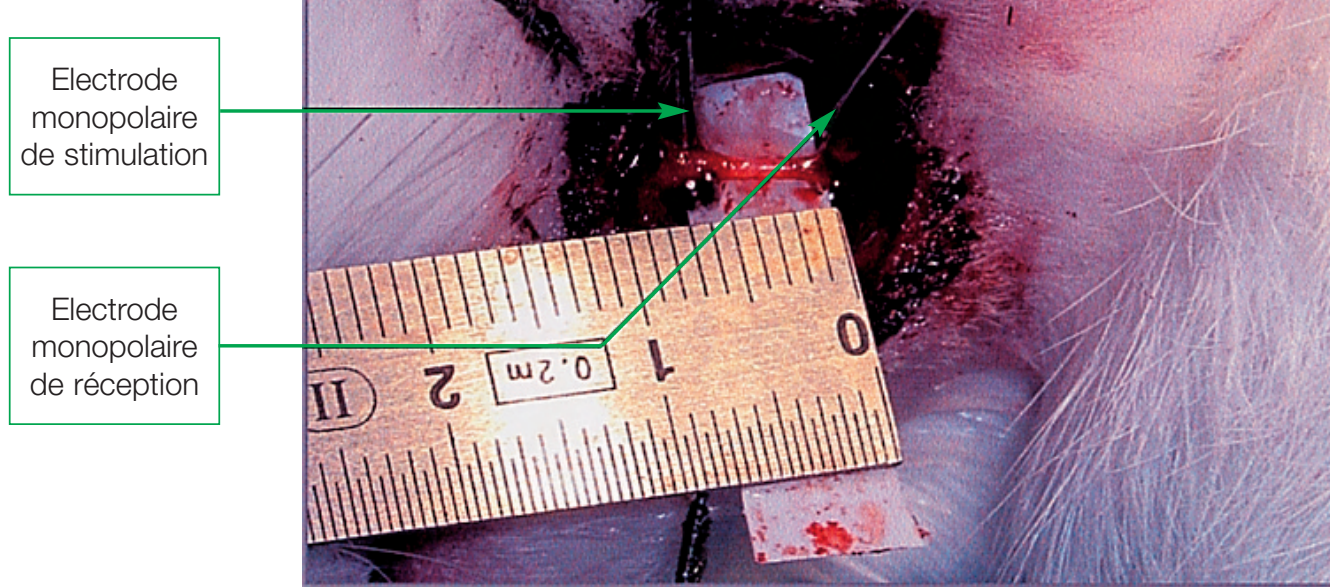

Tous les enregistrements étaient visualisés sur un oscilloscope. Après la réalisation du test électrophysiologique, le nerf était sectionné entre les 2 électrodes avec des ciseaux de microchirurgie. Cette section était franche et sans perte de substance. La suture des deux berges nerveuses a été effectuée sous microscope opératoire (ZEISS, SVG 11) :

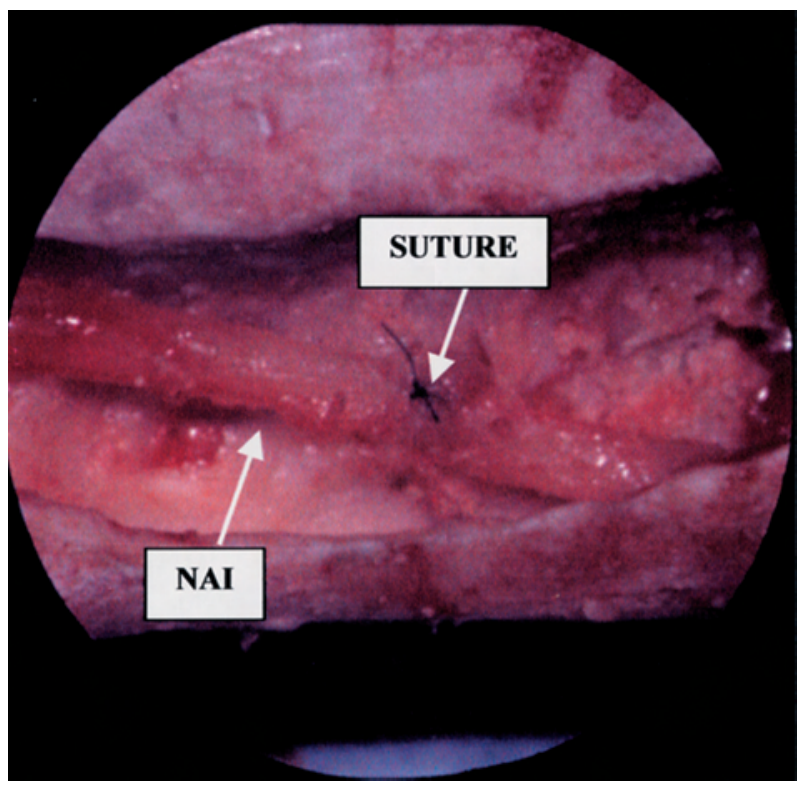

Figure 2 : Nerf alvéolaire inférieur suturé (DR) Sutured Inferior Alveolar Nerve (DR) suture épineuro-périneurale au fil nylon 10.0 et coaptation des deux berges sans tension (Fig. 2). Le nerf était repositionné dans son canal, puis les plans musculaires et cutanés étaient suturés. A $J+180$, une nouvelle intervention était pratiquée selon le même protocole pour exposer le NAI. Des tests électrophysiologiques étaient réalisées pour enregistrer les potentiels nerveux après régénération. L'électrode bipolaire de stimulation était placée en aval de la section proprement dite, et celle de réception en amont. Ces emplacements permettaient de contrôler la réalité de la réinnervation.

Après avoir déterminer l'intensité supraliminaire, différents stimuli étaient appliqués au nerf suturé:

1. - Stimulation électrique : détermination du seuil de sensibilité et étude de la réponse maximale.

2. - Stimulation mécanique de la zone mucogingivale physiologiquement innervé par le NAl.

L'animal était ensuite sacrifié et le NAI prélevé pour la réalisation d'une étude histologique. A J0, 2 prélèvements étaient effectués sur le nerf sain pour servir de référence histologique, et à $\mathrm{J}+180,4$ prélèvements étaient effectués sur le nerf suturé. Les prélèvements ont subi une médecine

buccale

chirurgie

buccale

VOL. $9, \mathrm{~N}^{\circ} 1$ 2003

page 23 
médecine

buccale

chirurgie

buccale

VOL. $9, \mathrm{~N}^{\circ} 1$ 2003

page 24 première préfixation dans le glutaraldéhyde $3,125 \%$ en tampon phosphate $0,1 \mathrm{M}$ pH 7.0 pendant 2 heures, puis deux lavages successifs en tampon phosphate $0,1 \mathrm{M} \mathrm{pH} 7.0$. Enfin, la fixation a été réalisée dans le tétroxyde d'osmium $1 \%$ en tampon phosphate $0,1 \mathrm{M} \mathrm{pH} 7.0$ pendant 1 heure. Les prélèvements fixés étaient ensuite deshydratés dans du bain d'acétone/eau, en concentration croissante d'acétone (1 minute à $30 \%, 1$ minute à $50 \%$, 20 minutes à $70 \%, 3$ fois 20 minutes à $90 \%, 4$ fois 20 minutes à $100 \%$ ). Les prélèvements ont subi ensuite 3 imprégnations en résine EMBED 812. D'abord dilué à $50 \%$ dans l'acétone pendant 1 heure, puis non dilué pendant 1 heure, et enfin non dilué pendant 1 heure à $37^{\circ} \mathrm{C}$. Les blocs obtenus étaient orientés puis polymérisés à $60^{\circ} \mathrm{C}$ pendant 24 heures. La première coupe semi-fine était réalisée au couteau de verre pour le repérage, la deuxième coupe semi-fine au diamant. Les coupes étaient colorées au bleu Azur II en milieu basique.

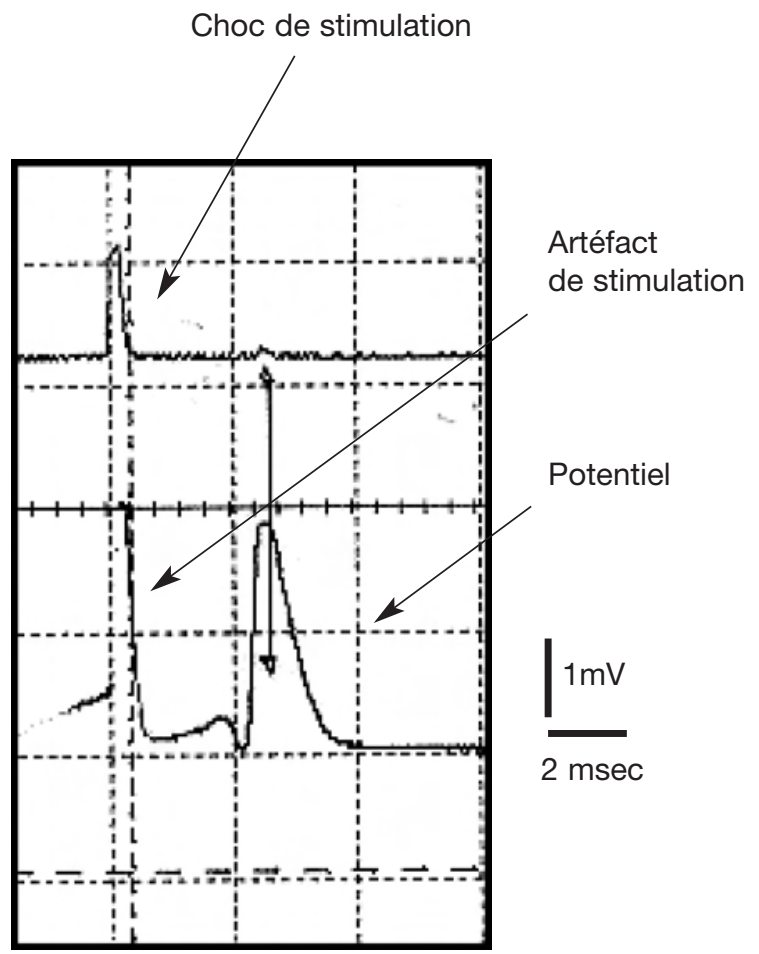

Figure 3 : Test électrophysiologique de référence Electrophysiological control test

\section{RÉSULTATS}

Dans cette étude, 8 lapins ont été opérés. La durée moyenne de chaque intervention, comprenant l'anesthésie, les enregistrements électrophysiologiques de référence, la section, la suture du nerf et la suture des différents plans avoisine les 120 minutes. Sur le plan électrophysiologique, 8 tracés de référence ont été réalisés. La figure 3 représente une des courbes obtenues. Cliniquement, une sensibilité nerveuse est observée dans 7 cas. L'obtention d'un potentiel nerveux global permet de confirmer la récupération fonctionnelle du nerf. $A J+180$, un nouveau potentiel d'action (Fig. 4) est observé chez 7 lapins; pour le $8^{\text {e}}$, il y a eu désunion de la suture nerveuse. Lorsqu'une stimulation à la sonde droite était réalisée dans le territoire d'innervation du NAI, un potentiel d'action était enregistré par l'électrode de réception située en amont de la section nerveuse.

Les observations histologiques ont montré des modifications quantitatives et qualitatives des différents échantillons nerveux. Les prélève-

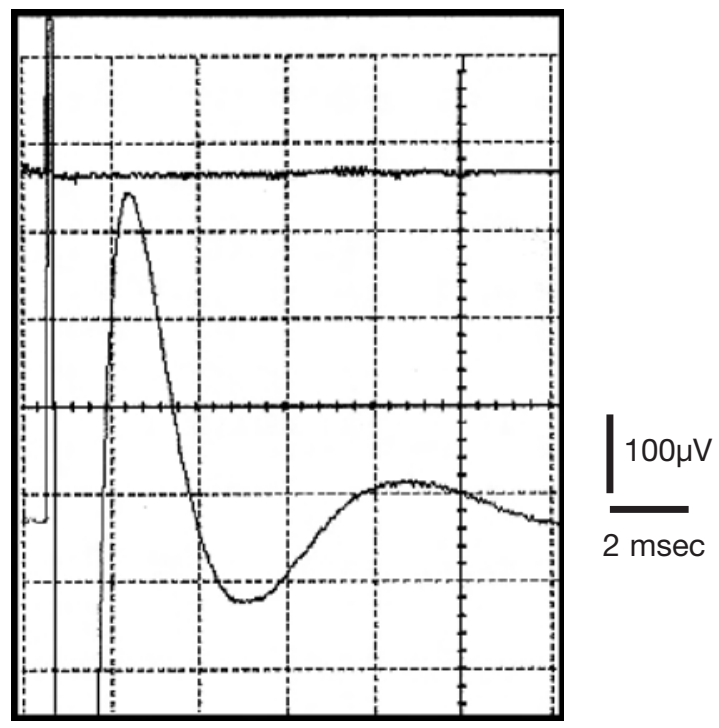

Figure 4 : Test électrophysiologique à 6 mois Electrophysiological test at 6 month 


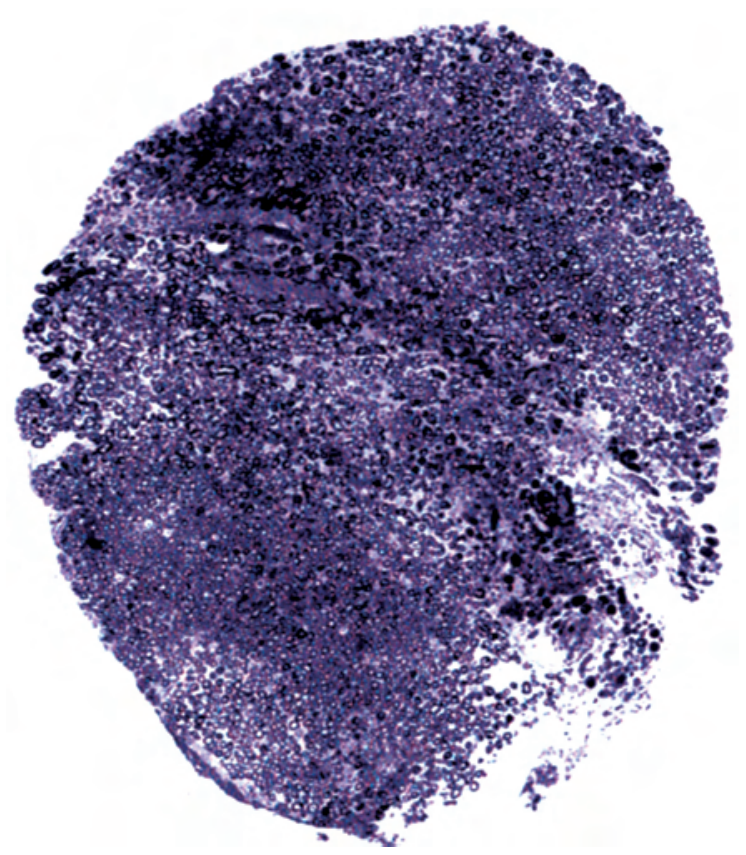

Figure 5 : Coupe histologique d'une section transversale d'un NAI saturé (DR)

Histological transverse section of sutured IAN (DR)

ments des nerfs suturés (Fig. 5) présentent en moyenne une diminution du nombre de fibres nerveuses par rapport aux nerfs témoins (Fig. 6). Les résultats montrent une diminution des fibres myélinisés de petits diamètres (de 2 à $7 \mu \mathrm{m}^{2}$ ) et une augmentation des fibres de grands diamètres (de 19 à $74 \mu \mathrm{m}^{2}$ ). Plus généralement, le diamètre moyen de ces fibres re-colonisées est sensiblement augmenté.

\section{DISCUSSION}

Le but de cette étude préliminaire était d'évaluer la régénération nerveuse du NAl chez le lapin après section chirurgicale sans perte de substance et suture conventionnelle. Seuls les dommages périphériques et non les mécanismes centraux ont été pris en compte.

Le NAI possède de plus quelques particularités physiologiques et anatomiques très spécifiques et celles-ci peuvent influencer la cicatrisation après le traumatisme. En effet, le NAl, branche de la Vème paire des nerfs crâniens, possède une proportion de fibres non myélinisées plus importantes qu'un bon nombre de nerfs d'ori-

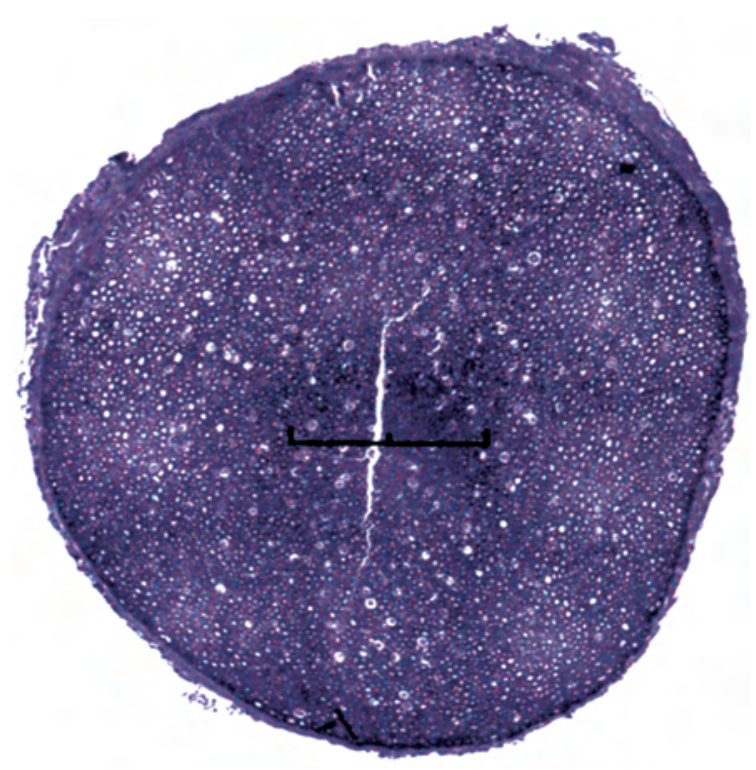

Figure 6 : Coupe histologique d'une section transversale d'un NAl contrôle (DR)

Histological transverse section of IAN control (DR)

gine spinale [13]. La position intracanalaire de ce nerf permet d'autre part de le protéger de certaines agressions mais le rend également vulnérable aux pressions qu'engendrent les oedèmes, les hématomes ou les fractures osseuses.

Dans cette étude sur des lapins, il était inconcevable d'utiliser les critères subjectifs décrits par Cornélius [12] en 1997, c'est pourquoi l'évaluation de la régénération nerveuse a été faite par la réalisation de tests éléctrophysiologiques (potentiels évoqués après stimulation électriques) et d'une étude histologique. Les 7 tests réalisés à $\mathrm{J}+180$ ont été positifs, à la fois par stimulation nerveuse mais également par stimulation du territoire sensitif correspondant.

Hasagawa [14] avait proposé des tests électrophysiologiques pour étudier la régénération du nerf radial du lapin avec et sans greffe, mais, ces tests $s$ 'effectuaient sur des fibres musculaires déclenchant ainsi une activité contractile. Tohyoma [15] en 1990, avait prélevé des nerfs de Japanese Wight Rabbits (JW) pour les transplanter sur des New-Zealand White Rabbits médecine

buccale

chirurgie

buccale

VOL. $9, \mathrm{~N}^{\circ} 1$ 2003

page 25 
médecine buccale chirurgie buccale

VOL. $9, \mathrm{~N}^{\circ} 1$ 2003

page 26
(NW) : il obtenait $10 \%$ de fibres régénérées après 6 semaines et $30 \%$ après 14 semaines. Nos résultats histologiques sont concordants avec ceux de Tohyoma : $52 \%$ de fibres histologiques recolonisées à $\mathrm{j}+180$.

Cependant, nos résultats ont été obtenus sur un nombre limité de lapins, avec des sections nerveuses franches, sans perte de substance, ni écrasement, suivies de sutures conventionnelles immédiates; les réactions tissulaires du nerf ont été ainsi limitées et la revascularisation précoce aussi facilitée. Or, selon Weiss et Taylor [16], il s'agit d'un point primordial dans la cicatrisation nerveuse.

L'utilisation d'un marqueur cellulaire telle la HRP (Horse Radish Peroxydase) devrait permettre dans des études ultérieures de quantifier le nombres de corps somatiques du ganglion trijéminal ayant dégénéré. En augmentant le nombre de lapins, différentes techniques de suture par laser CO2 $[17,18]$ ou par colle biologique Tissucol* par exemple [19,20], pourront être comparées. Dans ces différentes techniques, le temps d'intervention est généralement diminué car la technique consiste à

\section{RÉFÉRENCES}

1 - De Vicente JC, Lopez-Arranz JS. Preservation of the inferior alveolar nerve in the surgical approach to cancer of the posterior oral cavity. J Oral Maxillofac Surg 1998; 56: 1214-16.

2 - BAYS RA. Complications of orthognathic surgery (pp 193-221). WB Saunders, Philadelphia, 1997

3 - Nevearth EJ. Disabling complications following inadvertent overextension of a root canal filling material $J$ Endo 1989; 3: 135-9.

4 - Bovera M, Simion M, Rustici M, Misitano U. Hyatrogenic inferior alveolar nerve lesion during implant surgery. Italian Journal of Osseointegration 1994; 2: 7-14

5 - Sмith KG, Robinson PP. An experimental study of lingual nerve repair using epineural sutures or entabulation. Br J Oral Maxillofac Surg 1995; 33: 211-9.

6 - GHALI GE, JONES DL, WolfORD LM. Somatosensory evoked potential assessment of the inferior alveolar nerve following third molar extraction. Int $\mathrm{J}$ Oral Maxillofac Surg 1990;19: 18-21.

7 - Osborn TP, Frederickson G, SMall IA, Rogerson TS. A prospective study of complications related to the «souder» ou à «coller» les berges nerveuses. Cette étude avait également pour but d'informer le praticien sur le risque de certaines interventions chirurgicales menées sans analyse radiologique préalable et en méconnaissant l'anatomie du site concerné.

Les résultats obtenus après suture nerveuse ont été analysés dans le cadre d'un protocole expérimental. Les résultats préliminaires enregistrés au cours de cette étude sont très favorables et encourageants. Cependant, il s'agit ici d'une étude menée sur 8 lapins. L'utilisation de marqueur HRP devrait permettre de vérifier la conduction des fibres sensitives en appréciant le transport rétrograde axonal. Les séquelles de la section d'un nerf sont très invalidantes et souvent minimisées dans la littérature [21]. Les praticiens ne peuvent ignorer les possibilités de la microchirurgie et doivent en informer les patients : il est donc impossible en chirurgie dentaire de faire abstraction de ces nouvelles techniques de réparation nerveuse. La poursuite de cette recherche devrait aussi permettre d'améliorer les résultats voire d'améliorer la prise en charge des patients handicapés.

mandibular third molar. J Oral Maxillofac Surg 1985; 43: 767-9.

8 - Basbaum Al, Gautron M, Jazat $F$, Mayes $M$, GuILBAUD $G$. The spectrum of fibers loss in a model of neuropathic pain in the rat: an electron microscopic study. Pain 1991; 47: 359-67.

9 - Kiesselbach JE, Chamberlain JG. Clinical and anatomical observations on the relationship of the lingual nerve to the mandibular third molar region. J Oral Maxillofac Surg 1989; 42: 565-7.

10 - JONES RH. MICROSURGICAL REPAIR OF NERVES INJURED DURING third molar surgery. Aus Dent J 1992; 37: 253-61.

11 - RoBInson P. Observation on the recovery of sensation following inferior alveolar nerve injuries. $\mathrm{Br} J$ Oral Maxillofac Surg 1988; 26: 177-89.

12 - Cornelius CP, Roser M, Ehrenfeld M. Mikroneurale wiederherstellung nach iatrogen läsionen des $\mathrm{n}$. Lingualis und des $\mathrm{n}$. Alveolaris inferior. Mund Kiefer Gesichts Chir 1997; 213-223.

13 - HoLLAND GR. Fibre numbers and sizes in the inferior alveolar nerve of the cat. J Anat 1978; 127: 343-52. 
14 - Hasegawa J, Shibata M, TaKahashi H. Nerve coaptation studies with and without a gap in rabbits $\mathrm{J}$ Hand Surg 1996; 21: 259-65.

15 - TOHYAMA K, IDE C, OsaWA T. Nerve regeneration through the cryoinjured allogenic nerve graft in the rabbit. Acta Neuropathol 1990; 80: 138-44.

16 - WeISS P, TAYLOR AO. Further experimental evidence against «neurotropism » in nerve regeneration. J Exp Zool 1944; 95: 233.

17 - Huang T, Blanks R, CRumley R. Laser assisted nerve repair. Arch Otolaryngol Head Neck Surg 1992; 107 : 1150-4.

18 - Lauto A, Trickett R, Malik R, Dawes J, Owen E. Laser activated protein bands for peripheral nerve repair. Proc Laser Surg Spie 1995; 2363: 416-25.
19 - Curtis NJ, Lauto A, Trickett R, Owen E, Walter DM. Preliminary study of microsurgical repairs of the inferior alveolar nerve in rats using primary suturing and laser weld techniques. Int J Oral Maxillofac Surg 1998; 27: 476-81.

20 - Haussamen J, Schmelzeisen R. Current principles in microsurgical nerve repair $\mathrm{Br} J$ Oral Maxillofac Surg 1978; 34: 143-57.

21 - Ruggiero SL Trigeminal nerve injury and repair New York Dental Journal 1996; 36: 36-40

Remerciements au Professeur Falempin pour ses conseils et son aide et au Centre Commun d'Imagerie Cellulaire de I'USTL médecine

buccale

chirurgie

buccale

VOL. $9, \mathrm{~N}^{\circ} 1$

2003

page 27 